\title{
Retour d'expérience des attentats du 13 novembre 2015. Organisation des renforts par les Samu de province
}

\author{
Feedback on Terrorist Attacks on November 13, 2015. \\ Organization of Reinforcements by Provinces Medical Emergency Services
}

\author{
F. Braun · C. Ammirati - G. Auchères $\cdot$ M. Duché-Taillez $\cdot$ P. Goldstein $\cdot$ J. Jenvrin $\cdot$ V. Julié $\cdot$ P. Lévy-Chazal $\cdot$ \\ L. Nace $\cdot$ H. Roy $\cdot$ P. Valette $\cdot$ J. Miklin \\ Reçu le 12 janvier 2016; accepté le 13 janvier 2016 \\ (C) SFMU et Lavoisier SAS 2016
}

Résumé Les attentats parisiens du 13 novembre dernier ont mis en évidence la nécessité d'une organisation structurée de la réponse du système de santé dans laquelle la médecine d'urgence préhospitalière occupe une place prépondérante.

\author{
F. Braun $(\bowtie)$ \\ C. Ammirati \\ Samu 80, CHU Amiens \\ G. Auchères \\ Samu 45, CHR Orléans \\ M. Duché-Taillez \\ Samu 89, CH Auxerre \\ P. Goldstein \\ Samu 59, CHRU Lille \\ J. Jenvrin \\ Samu 44, CHU Nantes \\ V. Julié \\ Samu 28, CH Dreux \\ P. Lévy-Chazal \\ Samu 51, CHU Reims \\ L. Nace \\ Samu 54, CHRU Nancy \\ H. Roy \\ Samu 21, CHU Dijon \\ P. Valette \\ Samu 62, CH Arras \\ J. Miklin \\ Samu 76A, CHU Rouen
}

Structures de médecine d'urgence,

CHR Metz-Thionville, hôpital de Mercy, 1 allée du Château,

CS 45001, F-57085 Metz cedex 03

e-mail : fr.braun@chr-metz-thionville.fr
Au-delà de l'organisation francilienne, les Samu limitrophes ont rapidement engagé des moyens en renfort afin de sécuriser la réponse sanitaire dans l'hypothèse d'une augmentation $\mathrm{du}$ nombre de victimes. Cette stratégie opérationnelle repose sur des plans préétablis qu'il convient de réviser sur la base d'une entraide plus formalisée entre les Samu, principalement en province, où les équipes médicales sont moins nombreuses.

Mots clés Attaque terroriste · Organisation · Renforts · Urgence préhospitalière

\begin{abstract}
The Parisian terrorist attacks of last November 13, 2015 highlighted the need for a structured organization of the answer of the health system in which out-of-hospital emergency medicine occupies a dominating place. In addition to the Île-de-France organization, bordering medical emergency services quickly engaged reinforcements, in order to make safe the medical answer on the assumption of an increase in the number of victims. This operational strategy rests at the preestablished plans which are recommended to be updated on the basis of mutual assistance more formalized between the emergency medical services, mainly in the provinces where the medical teams are fewers.
\end{abstract}

Keywords Terrorist attacks · Organization · Reinforcements · Out-of-hospital emergency

\section{Introduction}

Les attentats du 13 novembre 2015 ont mis à l'épreuve notre système de santé [1]. Au-delà de la remarquable réponse des moyens préhospitaliers et hospitaliers d'Île-de-France [2] et des stratégies mises en place qui doivent être analysées, la solidarité immédiate exprimée par de nombreux Samu dans les régions limitrophes est à souligner car elle va 
probablement durablement modifier nos concepts opérationnels. Cet article présente le cadre réglementaire actuel des renforts sanitaires entre zones de défense, les grandes lignes de l'organisation de renforts des moyens médicalisés préhospitaliers mise en place, les choix stratégiques et les conclusions de ce retour d'expérience.

\section{Organisation des renforts sanitaires en France}

Actuellement, l'engagement de moyens sanitaires en renfort d'un département sur l'autre repose sur un cadre réglementaire récemment mis à jour par la création du dispositif ORSAN (ORganisation SANitaire) [3]. Dans tout le territoire national, la réponse à une situation d'exception, a fortiori sanitaire, est organisée au niveau de zones de défense et de sécurité chargées d'organiser la sécurité nationale et la défense civile et économique. La zone de défense est une circonscription administrative ayant à sa tête un représentant de l'État, le Préfet de zone. La métropole est divisée en sept zones de défense (Paris, Nord, Ouest, Sud-ouest, Sud, Sudest, Est), l'Outre-mer en cinq (Antilles, Guyane, Sud de l’Océan Indien, Nouvelle-Calédonie, Polynésie française) (Figs 1, 2).

Naturellement, le ministère de la Santé a calqué, sur ce découpage administratif, le dispositif de réponse sanitaire intégrant un volet sanitaire aux plans zonaux de défense et de sécurité [4] dont l'élaboration est sous la responsabilité du directeur général de l'Agence Régionale de Santé de zone. Ce plan zonal de mobilisation [5] identifie notamment :

- la répartition et les modalités de mobilisation des moyens matériels mobiles «tactiques » au sein de la zone. Ces moyens sont constitués des matériels et équipements mobiles dont sont dotés les Samu et Smur pour répondre aux situations sanitaires exceptionnelles (postes sanitaires mobiles, unités de décontamination...) ;

- les ressources humaines mobilisables en renfort ;

- les capacités de prise en charge des pathologies induites par l'exposition à un risque NRBC-E (nucléaire radiologique bactériologique chimique - explosif) ;

- les modalités de mobilisation des moyens d'autre zones ou de l'État (stocks stratégiques de produits de santé, réserve sanitaire);

- les plans de formation et d'entraînement des personnels de santé au sein de la zone.

- Le renforcement de l'offre de soins doit être décliné selon la cinétique de la crise, lente (épidémies, phénomènes climatiques prolongés...) ou rapide (catastrophes naturelles, accidents technologiques, attentats...). Dans le cadre des événements à cinétique rapide, "la réponse sanitaire repose prioritairement sur l'organisation de l'aide médicale urgente... mise en ouvre par le Samu territorialement compétent qui assure la régulation de l'événement. Dans la phase de réponse réflexe, ce dispositif de réponse doit naturellement s'appuyer sur l'assistance immédiate que les Samu contigus portent au Samu confronté à une situation grave, nécessitant une augmentation rapide de ses capacités de prise en charge des victimes. Ces dispositions d'assistance ont vocation à être formalisées dans le cadre d'un réseau, sans toutefois obérer leur caractère nécessairement spontané et immédiat » [5].

Le plan zonal de mobilisation pour faire face à une situation ayant un impact sanitaire exceptionnel est arrêté par le préfet de zone après avis du comité de défense de zone [6].

En complément de l'organisation zonale de la réponse sanitaire, le dispositif ORSAN (organisation de la réponse

\begin{tabular}{|c|c|c|c|}
\hline Nom & Siège & Ressort territorial & Préfet de zone \\
\hline Paris & Paris & Île-de-France & Préfet de police de Paris \\
\hline Nord & Lille & Nord-Pas-de-Calais, Picardie & $\begin{array}{l}\text { Préfet de Nord-Pas-de-Calais, } \\
\text { préfet du Nord }\end{array}$ \\
\hline Ouest & Rennes & $\begin{array}{l}\text { Basse-Normandie, Bretagne, Centre- } \\
\text { Val de Loire, Haute-Normandie, Pays } \\
\text { de la Loire } \\
\end{array}$ & $\begin{array}{l}\text { Préfet de Bretagne, préfet d'Ille- } \\
\text { et-Vilaine }\end{array}$ \\
\hline \begin{tabular}{|l|} 
Sud- \\
Ouest
\end{tabular} & Bordeaux & $\begin{array}{l}\text { Aquitaine, Limousin, Midi-Pyrénées, } \\
\text { Poitou-Charentes }\end{array}$ & $\begin{array}{l}\text { Préfet d'Aquitaine, préfet de la } \\
\text { Gironde }\end{array}$ \\
\hline Sud & Marseille & $\begin{array}{l}\text { Corse, Languedoc-Roussillon, } \\
\text { Provence-Alpes-Côte d'Azur }\end{array}$ & $\begin{array}{l}\text { Préfet de Provence-Alpes-Côte } \\
\text { d'Azur, préfet des Bouches-du- } \\
\text { Rhône }\end{array}$ \\
\hline $\begin{array}{l}\text { Sud- } \\
\text { Est }\end{array}$ & Lyon & Auvergne, Rhône-Alpes & $\begin{array}{l}\text { Préfet de Rhône-Alpes, préfet du } \\
\text { Rhône }\end{array}$ \\
\hline Est & Metz & $\begin{array}{l}\text { Alsace, Bourgogne, Champagne- } \\
\text { Ardenne, Franche-Comté, Lorraine }\end{array}$ & $\begin{array}{l}\text { Préfet de Lorraine, préfet de la } \\
\text { Moselle }\end{array}$ \\
\hline
\end{tabular}

Fig. 1 Les zones de défense en de sécurité en métropole. Les couleurs correspondent à celles de la Fig. 2 


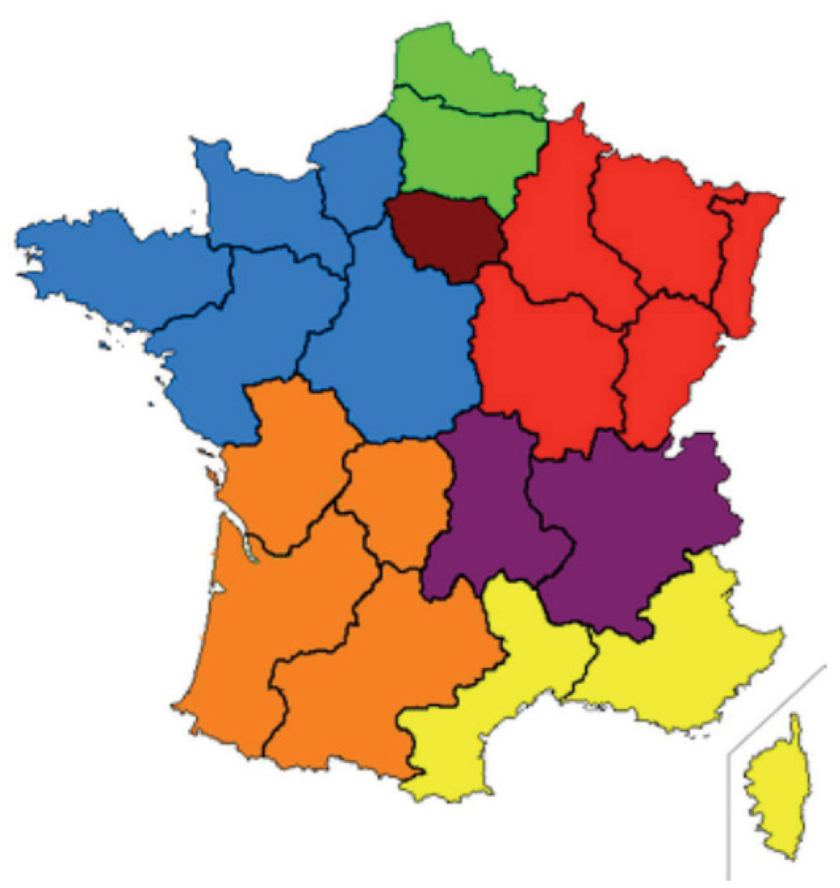

Fig. 2 Zones de défense et de sécurité en France métropolitaine. Les principaux éléments sont repris dans la Fig. 1

du système de santé en situations sanitaires exceptionnelles) formalise la coordination des dispositifs existants dans les trois secteurs sanitaires, ambulatoire (la médecine libérale), hospitalier et médicosocial. Ce dispositif comprend cinq volets répondant à des situations sanitaires particulières :

- ORSAN AMAVI : accueil massif de victimes non contaminées ;

- ORSAN CLIM : prise en charge de nombreux patients suite à un phénomène climatique ;

- ORSAN EPI-VAC : gestion d'une épidémie ou pandémie sur le territoire national ;

- ORSAN BIO : prise en charge d'un risque biologique connu ou émergent ;

- ORSAN NRC : prise en charge d'un risque nucléaire, radiologique, chimique.

La notion de situation sanitaire exceptionnelle (SSE) définie dans ce plan englobe toutes les situations susceptibles d'engendrer une augmentation de la demande de soins ou de perturber l'offre de soins. Ce concept témoignant d'un déséquilibre entre « la demande » et « l'offre » qui aboutit à un débordement, même transitoire, des structures médicales immédiatement disponibles, est depuis longtemps le fondement de la médecine de catastrophe [7].

Le dispositif ORSAN vient naturellement en complément du dispositif ORSEC (Organisation de la réponse de sécurité civile), les deux pouvant être mis en œuvre séparément ou concomitamment. En cas de nombreuses victimes, l'articu- lation opérationnelle entre les mesures prises au titre du dispositif ORSEC et du schéma ORSAN est assurée par le Samu. Il est proposé de retenir le critère de $25 \%$ d'urgences absolues (UA) et $75 \%$ d'urgences relatives (UR) pour établir le dimensionnement des personnes à prendre en charge. Ce chiffre pouvant bien entendu être modifié en fonction des risques identifiés. La prise en charge des victimes médicopsychologiques, quant à elle, fait partie intégrante du dispositif d'aide médicale urgente et doit être intégrée à la démarche de planification.

Le dispositif ORSAN précise également la mission du Samu de zone qui, dans le cadre du plan zonal de mobilisation, apporte un appui technique à l'ARSZ (agence régionale de santé de zone) et, lors d'une SSE, renforce les Samu directement impactés en :

- mobilisant les moyens tactiques de la zone ;

- mettant en œuvre les modalités de renfort en personnels de santé ;

- proposant l'intégration de renforts venant d'autres zones ;

- assurant la synthèse des moyens disponibles ;

- coordonnant les hélicoptères sanitaires ;

- tenant informé(s) le(s) Samu territorialement compétent (s) de la prise en charge et de l'orientation des victimes.

Actuellement, aucun texte réglementaire ne définit clairement quel Samu est désigné comme Samu de zone, cette désignation étant effectuée par l'ARSZ. Un projet de décret en conseil d'État prévoit une modification de l'article R3131-9 de Code de la Santé Publique afin que cette désignation soit normalisée.

\section{Description de l'organisation opérationnelle des Samu extérieurs à l'Île-de-France}

À $21 \mathrm{~h} 20$, la première explosion retentit au Stade de France et, à partir de $21 \mathrm{~h} 25$, le Samu-Centre 15 de Paris prend en charge plusieurs appels pour des fusillades au cœur de la capitale. Avant même la transmission de l'alerte aux zones de défenses proches de Paris, de nombreux Samu ont anticipé la demande, informés de la situation (attentats multisites) directement par les médias d'information continue. Cette information, rapidement vérifiée auprès du Samu de Paris, a permis la mise en alerte très précoce de moyens médicaux préhospitaliers et leur pré-positionnement « aux portes de la capitale ». Une heure après la première explosion au Stade de France, le Samu du Nord se met en configuration zonale et anticipe l'envoi de moyens de renfort en alertant les Samu 80, 62, 60 et 02. Parallèlement, plusieurs autres Samu anticipent spontanément l'engagement d'équipes Smur en renfort, puis rapidement de moyens de cellules d'urgence médicopsychologique (CUMP) (Tableau 1). Les distances entre les villes concernées et Paris ainsi que les 
Tableau 1 Moyens engagés dans la nuit du 13 au 14 novembre 2015 par les différents Samu extérieurs à la zone de défense et de sécurité d'Île-de-France.

\begin{tabular}{|c|c|c|c|c|c|}
\hline & Alerte & Via & UMH & HéliSmur & CUMP \\
\hline Samu 59 & $22 h$ & Médias & 2 & 1 & oui \\
\hline Samu 62 & $22 h 30$ & Samu 59 & 1 & 0 & non \\
\hline Samu 80 & $22 h 38$ & Samu 59 & 1 & 1 & non \\
\hline Samu 57 & $22 h 40$ & Médias & 0 & 0 & oui \\
\hline Samu 54 & $22 h 45$ & Samu 57 & 0 & 1 & non \\
\hline Samu 44 & $22 h 45$ & Médias & 0 & 1 & non \\
\hline Samu 89 & $23 h 12$ & Codis 89 & 0 & 1 & non \\
\hline Samu 51 & $23 h 17$ & Samu 54 & 1 & 1 & non \\
\hline Samu 21 & $23 h 50$ & Samu 75 & 0 & 1 & non \\
\hline Samu 45 & $23 h 58$ & Samu 75 & 1 & 1 & oui \\
\hline Samu 28 & $00 h 03$ & Samu 75 & 2 & 1 & non \\
\hline Samu 76A & $23 h 46$ & Samu 75 & 1 & 1 & non \\
\hline
\end{tabular}

délais d'acheminement des HéliSmur sont précisés en annexe (Fig. 3).

L'engagement de ces moyens supplémentaires, à la demande du Samu de Paris, répondait à une logique opérationnelle claire : la capacité de la salle de spectacle du Bataclan étant d'un millier de personnes, l'hypothèse minimale d'une centaine de patients UA supplémentaires était raisonnable. Ce nombre de victimes potentielles aurait, de manière évidente, saturé le système hospitalier francilien et justifiait d'envisager des prises en charge dans des établissements plus lointains, voire au minimum des transferts de patients après une première prise en charge chirurgicale d'hémostase. L'assaut par les forces de l'ordre de la salle de spectacle permettait malheureusement de découvrir de très nombreuses personnes décédées, victimes de lésions balistiques soit immédiatement mortelles, soit n'ayant pu être prises en charge rapidement car inaccessibles.

Parallèlement, à $21 \mathrm{~h} 45$, le centre opérationnel de réception et de régulation des urgences sanitaires et sociales (CORRUSS) du ministère de la Santé était activé en centre de crise sanitaire (niveau 3) par le Directeur Général de la Santé [8]. Un premier message d'alerte rapide sanitaire (MARS) était adressé à minuit à tous les établissements disposant d'une structure de médecine d'urgence les invitant à se mobiliser en cas d'autres attaques et plusieurs conférences téléphoniques étaient organisées avec les Samu de zone.

Une fois la situation stabilisée, le Samu de Paris et le CORRUSS contactaient, entre $1 \mathrm{~h} 45$ et $2 \mathrm{~h}$, l'ensemble des Samu engagés pour annuler les renforts Smur, les moyens d'île-de-France étant alors suffisants.
L'analyse de cette phase initiale permet de relever des points intéressants.

\section{L'alerte}

La transmission de l'alerte aux différents Samu a suivi des circuits très différents (Fig. 1) : information transmise par les médias et vérifiée auprès du Samu de Paris (Lille, Nantes et Metz), alerte via le Samu zonal (Amiens et Arras), un autre Samu (Nancy), via les sapeurs-pompiers (Auxerre) ou enfin demande directe du Samu de Paris (Dijon, Dreux, Rouen et Orléans). Lors de cette crise, nous devons constater que la « veille » effectuée par certaines plateformes de régulation médicale sur les médias d'information a permis une activation plus précoce que les circuits d'alerte plus formalisés.

\section{L'engagement et la gestion des moyens}

Tous les moyens engagés, Smur, HéliSmur et cellules d'urgence médicopsychologique (CUMP) l'ont été à la demande du Samu de Paris. Les points de rassemblement des moyens ont été définis et précisés aux intervenants avant leur départ : hôpital Necker pour les ambulances de réanimation (AR), aéroport d'Issy-les-Moulineaux pour les HéliSmur, mairie $\mathrm{du} \mathrm{XI}^{\mathrm{e}}$ arrondissement (CUMP 59) et hôpital de la PitiéSalpêtrière (CUMP 57). L'engagement des AR et des CUMP n'a pas posé de problème particulier mais celui des HéliSmur appelle quelques remarques. Quatre Samu (89, 51, 28 et 62) disposent d'un HéliSmur pour lequel l'activité est limitée en journée. Pour deux d'entre eux, le besoin d'une réquisition préfectorale autorisant la mission a été nécessaire, 


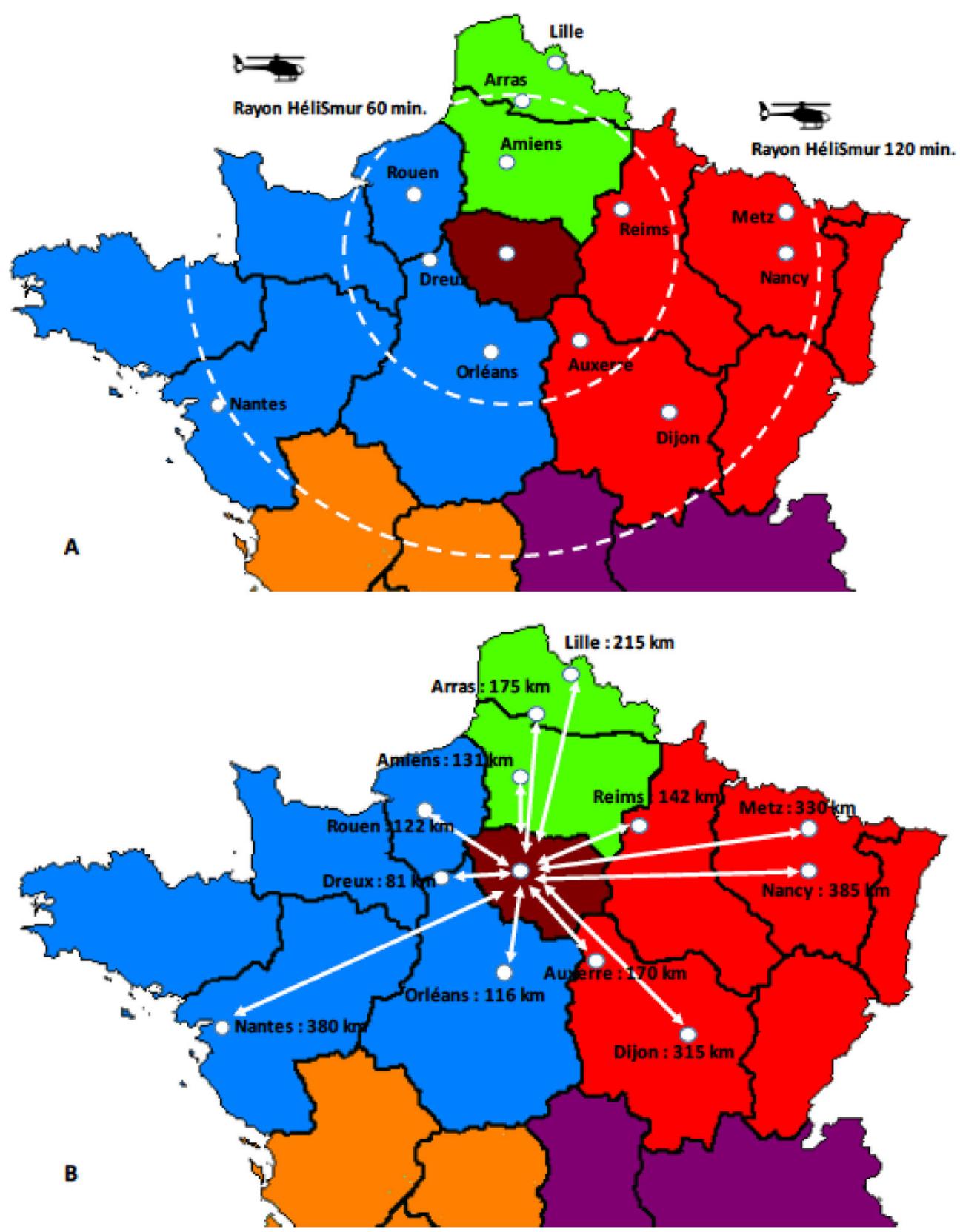

Fig. 3 : A : délais d'intervention des HéliSmurs engagés par les Samu-Centre 15 de province ; B : distance routière entre les villes des Samu-Centre 15 ayant engagé des moyens de renfort et Paris

réquisition génératrice de perte de temps. Dans un tel contexte de sécurité renforcée, il est également indispensable que les HéliSmur soient identifiés et donc que leur immatriculation soit connue et transmise avant leur engagement.

Concernant l'annulation des moyens, celle-ci a été rapidement et efficacement conduite par le Samu de Paris et le CORRUSS permettant un retour rapide des moyens engagés vers leur base.

Si tous les Samu engagés soulignent la rapidité avec laquelle ils ont pu mobiliser du personnel en renfort, la difficulté de savoir « qui décide » est relevée par tous. La néces- sité d'avoir un interlocuteur unique apparaît comme évidente. Si les informations transmises par les Samu de Paris et de Lille (zonal) étaient rapides et pertinentes, plusieurs Samu soulignent le manque de pertinence d'informations demandées tardivement par les Samu et ARS de zone, les moyens étant déjà engagés, voire sur le retour.

Notons enfin que les moyens engagés par ses différents Samu étaient bien des moyens supplémentaire ne les mettant pas en difficulté pour assurer les missions habituelles, les transferts médicalisés entre hôpitaux étant, lorsque cela était possible, reportés. 


\section{Discussion}

La nature brutale, multisite, générant de très nombreuses victimes et surtout évolutive (entre $21 \mathrm{~h} 30$ et $1 \mathrm{~h}$ ) de cette crise nous impose des réflexions sur la réponse sanitaire déployée, notre organisation nationale et l'indispensable anticipation devant être mise en place en province [9].

Pour gérer cette crise, le Samu de Paris a pu compter sur 45 équipes Smur sur le terrain et 9 HéliSmur à disposition. Vingt-cinq équipes étaient en attente pour assurer les missions habituelles mais aussi pour répondre à une escalade des besoins. À titre d'exemple, la région Lorraine (2,4 millions d'habitants) dispose de 24 équipes Smur et d'un HéliSmur !

Cette simple constatation comptable met en évidence l'impérieuse nécessité, en cas d'attentat similaire en région, d'engager au plus vite des moyens préhospitaliers supplémentaires provenant des régions ou des pays limitrophes. Dans ce contexte, l'hélicoptère est un moyen sûr et rapide pour acheminer des renforts.

Cette saturation, prévisible, du système préhospitalier en province doit aussi être anticipée au niveau national pour des patients particuliers, enfants, brûlés, intoxiqués relevant de thérapeutiques spécialisées comme l'oxygénothérapie hyperbare...

L'évolution de nos concepts et l'amélioration de notre organisation doivent être une conséquence de cette crise. Quatre axes d'amélioration doivent être envisagés afin de proposer une organisation efficiente et lisible.

\section{Alerte, recherche et diffusion de l'information}

L'interconnexion entre les plateformes d'appels des secours (sapeurs-pompiers, forces de l'ordre) et des soins d'urgence (Samu-Centre 15) est la règle sur tout le territoire national. $\mathrm{Si}$ des efforts doivent être faits pour améliorer les outils de communication, les échanges sont opérationnels et efficaces. Nous avons constaté, lors de cette crise, l'importance des réseaux " parallèles » d'information et en particulier les médias d'information continue. Il apparaît donc essentiel, dans un souci de rapidité et de transmission de l'alerte que les Samu-Centre 15, ou au moins ceux à vocation régionale et/ou zonale, assurent une veille de ces réseaux.

Le Samu en charge de la crise doit immédiatement informer les Samu limitrophes et le Samu référent zonal (SRZ) afin qu'un engagement de renforts soit le plus rapide possible. L'anticipation doit, à ce stade, être la règle.

Passé cette phase initiale, le SRZ, organisé en gestion de crise, est en appui du Samu territorialement compétent pour assurer la recherche et la diffusion de l'information aux autres Samu zonaux, au CORRUSS, et anticiper les renforts extrazonaux sur la base d'un découpage anticipé du territoire national comparable à celui utilisé en Île-de-France et communément appelé « Plan Camenbert» [10].

L'information des ARS et en particulier de l'ARSZ est essentielle dans la gestion de crise à cinétique plus lente mais n'apporte pas de plus-value opérationnelle à cette phase de la crise. Il semble alors pertinent que l'information des ARS soit un des rôles dévolus au CORRUSS.

\section{Stratégie(s) d'intervention immédiate}

La nature multisite du risque et la possibilité de sur-attentat (explosion par exemple d'un véhicule piégé à proximité des équipes sur place) justifient, comme l'a montré le Samu de Paris, un engagement raisonné des moyens. Si pour l'Îlede-France, cet engagement raisonné repose sur le maintien d'équipes « en attente », cette stratégie n'est pas applicable en province, les effectifs immédiatement opérationnels étant plus faibles.

La stratégie d'un engagement immédiat de moyens de renfort par les autres départements, selon une logique centripète, est ici évidente et repose bien sur les Samu limitrophes comme le précise le principe du plan zonal de mobilisation. Les Samu concernés doivent disposer alors d'une totale autonomie dans l'engagement des moyens sanitaires à leur disposition, quitte bien sûr à en informer le SRZ qui se charge d'organiser le maintien de la couverture des risques, par exemple en redéployant les postes sanitaires mobiles (PSM).

Cette logique de renforts immédiats impose, à chaque Samu, d'identifier dès maintenant un ou des points de rassemblement des moyens (PRM) pour les véhicules mais également les HéliSmur.

Si l'engagement immédiat de moyens Smur est habituel, constatons que l'engagement des moyens de la CUMP est souvent, et à tort, retardé : dès le 13 novembre, 300 personnes ont été prises en charge par les équipes d'urgence psychiatrique d'Île-de-France [11] ! Notre stratégie d'engagement doit donc, désormais, intégrer le déclenchement immédiat de la CUMP locale mais aussi celui de renforts dans la même logique que les équipes de réanimation préhospitalière.

Dans la stratégie à mettre en œuvre, nous avons pu constater, lors des attentats du 13 novembre dernier, le peu d'intérêt à identifier les lits d'hospitalisation disponibles. En effet, dans un tel contexte, il est plus nécessaire d'identifier les plateaux opératoires opérationnels que les lits d'hospitalisation, fussent-ils de réanimation ou de soins intensifs. Les Plans Blancs des établissements de santé doivent prendre en compte cette donnée nouvelle.

\section{Engagement et suivi des moyens de renfort}

L'engagement de moyens sanitaires par les Samu limitrophes doit être le plus rapide possible. Ces moyens, regroupés 
au niveau d'un PRM, sont mis à disposition du Samu territorialement compétent qui les gère comme ses propres moyens. Informé de ces engagements, le SRZ organise la couverture sanitaire des zones Smur possiblement démunies en concertation avec les Samu concernés. Cette situation doit être anticipée dans le cadre des plans zonaux de mobilisation en autorisant bien entendu des couvertures interzonales.

Il semble important que la totalité des moyens en renfort soient sous l'autorité du Samu « de première ligne » dès lors qu'ils sont engagés. La gestion, le 13 novembre, de ces moyens par le seul Samu de Paris a permis leur libération rapide dès lors qu'ils n'étaient plus nécessaires. Il est donc indispensable que du personnel soit entièrement dédié à cette gestion.

Une vision globale de l'engagement des moyens sanitaire doit être à la disposition du CORRUSS, régulièrement informé par les SRZ.

Brider cette autonomie de décision des Samu ne pourrait avoir que des conséquences dramatiques en retardant l'engagement de moyens sanitaires indispensables. En cas d'incertitude quant aux besoins initiaux, le positionnement de moyens de renfort en limite de département assure une couverture rapide en cas de crise évolutive.

Concernant les moyens héliportés, il est indispensable que les HéliSmur n'ayant pas d'autorisation permanente de vols puissent obtenir par un circuit extrêmement rapide cette autorisation préfectorale. La proximité du SRZ avec la préfecture de zone pourrait faciliter l'obtention de cette autorisation. Possiblement utilisés dans des " missions santé », la place des hélicoptères d'état dans ces situations de crise doit être précisée quant à la priorité de leur mission, de sécurité ou sanitaire.

\section{Répertoire et suivi des victimes}

L'identification et le suivi des victimes restent une problématique habituelle lors d'événement à fort impact sanitaire. Développé en Île-de-France, le système SINUS (Système d'information numérique standardisé) [12], permet un tel suivi. En cours de développement sur tout le territoire national, il permettra rapidement une gestion globale, uniforme, des victimes, à condition que tous les intervenants en soient équipés, et particulièrement les structures des urgences amenées à prendre en charge des victimes se présentant spontanément dans les services. Une réflexion doit aussi rapidement être conduite afin que ce système soit compatible avec l'identification des victimes au sein des systèmes d'information des Samu-Centre 15 mais aussi des systèmes d'information hospitaliers.

Dès lors que la gestion de la crise dépasse les limites d'une seule zone de défense, le CORRUSS doit pouvoir gérer les listes de victimes.

\section{Gestion du « post-immédiat »}

Passée cette phase d'engagement immédiat des moyens sanitaires, la mise en œuvre de moyens nationaux doit être conduite sous l'autorité du Directeur Général de la Santé s'appuyant sur le CORRUSS et les ARS. S'appuyant déjà sur le réseau des établissements de santé de référence [13] pour la gestion sanitaire des risques NRBC-E, il semble intéressant que, dès la phase immédiate et plus pour des réflexions stratégiques que relevant des prises en charge sanitaires, le CORRUSS puisse faire immédiatement appel à des experts issus de la médecine d'urgence afin d'apporter une vision plus « opérationnelle» de la situation. Le Conseil National de l'Urgence Hospitalière [14] pourrait utilement proposer une telle liste d'experts à la Direction Générale de la Santé. Cette configuration permettra la gestion d'une crise à cinétique plus longue ou nécessitant des moyens qui dépassent les possibilités sanitaires habituelles. Dans ce contexte, l'articulation avec les moyens du service de santé des Armées, décidée au niveau national, doit pouvoir être anticipée et déclinée au niveau local.

La gestion du " post-immédiat » doit également intégrer la remise en état du système de santé en assurant le plus rapidement possible un fonctionnement normal des établissements de santé. L'appel à la réserve sanitaire [15], gérée par l'établissement de préparation et de réponse aux urgences sanitaires (EPRUS), doit lui aussi être anticipé afin de répondre aux demandes des hôpitaux.

Enfin, la gestion du " post-immédiat » doit prendre en compte la prise en charge médicopsychologique des soignants directement impliqués dans la gestion de cette crise. Trop souvent négligée, cette prise en charge réalisée par les CUMP, sans être imposée, doit être fermement proposée à des professionnels se croyant souvent « invulnérables ».

\section{Conclusion}

La rapidité d'analyse et de décision des Samu-Centre 15, leur naturelle capacité à anticiper l'évolution d'une situation sanitaire, fût-elle exceptionnelle, font de la médecine d'urgence préhospitalière l'élément essentiel de la réponse immédiate à une situation de crise sanitaire. Notre organisation nationale, basée sur une analyse médicale préalable - la régulation médicale -, une médicalisation préhospitalière spécialisée et une filiarisation des prises en charge, autorise une réponse adaptée à tout problème de santé. Pour autant, faire face à une série d'attentats simultanés, a fortiori en dehors de la région parisienne, impose d'adapter nos concepts de prise en charge et notre stratégie opérationnelle.

L'autonomie de décision opérationnelle des Samu-Centre 15 doit être réaffirmée et probablement élargie. Le réseau des Samu doit désormais être reconstruit dans une logique 
opérationnelle à l'échelle d'une région et/ou d'une zone de défense en se basant sur des Samu référents capables de répondre à un cahier des charges précis.

«L'expérience de chacun est le trésor de tous » (Gérard de Nerval) : sachons profiter de cette expérience trop chèrement acquise pour adapter nos moyens de réponse sanitaire. La santé, et en son sein la médecine d'urgence, sont des éléments essentiels de la résilience de la société aux attentats.

Liens d'intérêts : Les auteurs déclarent ne pas avoir de lien d'intérêt.

\section{Références}

1. Haug C (2015) Report from Paris. N Engl J Med 373:2589-93

2. Hirsch M, Carli P, Nizard R, et al (2015) The medical response to multisite terrorist attacks in Paris. Lancet 386:2535-8

3. Direction générale de la Santé. Ministère de la Santé (2015) Guide d'aide à l'organisation de l'offre de soins en situations sanitaires exceptionnelles. http://www.sante.gouv.fr/le-dispositiforsan (Dernier accès le 5 janvier 2016)

4. Le Ministre de l'intérieur, la Ministre des affaires sociales et de la santé (2013) Circulaire interministérielle $\mathrm{N}^{\circ}$ DGS/DUS/DGSCGC/ 2013/374 du 26 septembre 2013 relative à l'élaboration du plan zonal de mobilisation des ressources sanitaire. http://circulaire. legifrance.gouv.fr/pdf/2013/11/cir 37645.pdf (Dernier accès le 5 janvier 2016)

5. Le Premier ministre (2013) décret $n^{\circ}$ 2013-15 du 7 janvier 2013. http://www.legifrance.gouv.fr/affichTexte.do?cidTexte=JORFTE XT000026915951 (Dernier accès le 5 janvier 2016)

6. 2015) Article R.1311-25 de code de la défense. http://www. legifrance.gouv.fr/affichCodeArticle.do?cidTexte=LEGITEXT00
0006071307\&idArticle=LEGIARTI000006574248\&dateTexte $=$ $\&$ categorieLien $=$ cid (Dernier accès le 5 janvier 2016)

7. Noto R, Huguenard P, Larcan A (1987) Médecine de catastrophe. Masson éditeur, Paris

8. Philippe JM, Brahic O, Carli P, et al (2016) French Ministry of Health's Response to Paris Attacks of $13^{\text {th }}$ November (2015). Crit Care [in press]

9. Kellermann A, Peleg K (2013) Lessons from Boston. N Engl J Med 368:1956-7

10. Carli P, Nahon M. RETEX attentats terroristes. 13 novembre 2015 (2015) $5^{\mathrm{e}}$ journée de régulation médicale. Paris. http://www.samude-france.fr/documents/actus/155/820/33 jsudf 151217 retex prehosp_alerte_s75_carli.pdf (Dernier accès le 4 janvier 2016)

11. Dantchev N, Ben Younes S, Mullner J, et al (2016) Retour d'expérience des attentats du 13 novembre 2015. Prise en charge psychologique hospitalière des impliqués. Ann Fr Med Urg 6:55-61

12. Le Ministre de l'intérieur (2010) Arrêté du 17 février 2010 portant création d'un traitement automatisé de données à caractère personnel dénommé "système d'information numérique standardisé » (SINUS) http://www.legifrance.gouv.fr/eli/arrete/2010/2/ 17/IOCD0930321A/jo/texte (Dernier accès le 5 janvier 2016)

13. Le Ministre de la santé et des solidarités (2005) Arrêté du 30 décembre 2005 relatif à la liste des établissements de santé de référence. http://www.legifrance.gouv.fr/affichTexte.do?cidTexte=JORFTEXT000000636116 (Dernier accès le 5 janvier 2016)

14. Le Premier ministre (2012) Décret $N^{\circ} 2012-1138$ du 9 octobre 2012 relatif au conseil national de l'urgence hospitalière. http:// www.legifrance.gouv.fr/affichTexte.do?cidTexte=JORFTEXT0000 26476964\&categorieLien=id (Dernier accès le 5 janvier 2016)

15. 2007) Loi $n^{\circ} 2007-294$ du 5 mars 2007 relative à la préparation du système de santé à des menaces sanitaires de grande ampleur. http://legifrance.gouv.fr/affichTexte.do?cidTexte=JORFTEXT000000461266 (Dernier accès 5 janvier 2016) 